\title{
Three-Dimensional Nasolabial Displacement during Movement in Repaired Cleft Lip and Palate Patients
}

\author{
Carroll-Ann Trotman, B.D.S., M.A., M.S., Julian J. Faraway, Ph.D., and Greg K. Essick, D.D.S., Ph.D. \\ Chapel Hill, N.C., and Ann Arbor, Mich.
}

\begin{abstract}
The objective of this study was two-fold: (1) to explore the suitability of a novel modified Procrustes fit method to adjust data for head motion during instructed facial movements, and (2) to compare the adjusted data among repaired unilateral $(n=4)$ and bilateral $(n=5)$ cleft lip and palate patients and noncleft control subjects $(n=50)$. Using a video-based tracking system, three-dimensional displacement of 14 well-defined nasolabial landmarks was measured during four set facial animations without controlling for head motion. The modified Procrustes fit method eliminated the contributions of head motion by matching the most stable landmarks of each video-recorded frame of the face during function to frames at rest. Its effectiveness was found to approximate that of a previous method (i.e., use of a maxillary occlusal splint to which stable dentition-based markers were attached). Data from both the unilateral and bilateral cleft lip and palate patients fell outside the normal range of maximum displacements and of asymmetry, and individual patients demonstrated greater right-versus-left asymmetry in maximum displacement than did individual noncleft subjects. It is concluded that the modified Procrustes fit method is fast, is easy to apply, and allows subjects to move the head naturally without the inconvenience of a splint while facial movement data are being collected. Results obtained using this method support the view that facial movements in cleft patients may be severely hampered and that assessment of facial animation should be strongly considered when contemplating surgical lip revisions. (Plast. Reconstr. Surg. 105: 1273, 2000.)
\end{abstract}

To even a casual observer, the most obvious disfigurement of the cleft face is seen around the nasolabial region; despite significant improvements in surgical repair, considerable soft-tissue distortions are common. Most previous methods of assessing the quality of cleft repairs have been based on either subjective clinical impression ${ }^{1,2}$ or two-dimensional objective measures of nasolabial disfigurement and asymmetry at rest. ${ }^{3-6}$ Large interrater variation, however, limits the validity of subjective assessments of an individual's facial movements, particularly as surgical corrections are likely to result in small incremental improvements rather than dramatic changes. ${ }^{2}$ Also, any assessment of the quality of surgical reconstruction should include dynamic (i.e., the face during function) as well as static (face during rest) measures. ${ }^{2,3,7}$ To these ends, we have developed a novel method that captures facial movement with sufficient sensitivity to allow an objective analysis of dynamic outcomes of cleft surgeries. $^{8-10}$

In our method, small spherical markers are attached to specific landmarks on the face, and video recordings are made during a series of standardized animations, during which the head remains unrestrained. To estimate the true three-dimensional displacements of facial landmarks from rest, the contributions of head motion must be subtracted because they add to and confound measurements of the movement of the facial landmarks. To estimate the contributions of head motion, stable reference markers on the craniofacial region can be tracked (e.g., markers referenced to the dentition by the use of a maxillary occlusal splint). This method, employed in our previous work, is cumbersome and may alter patients' movement of the face, particularly within the upper lip region.

In part 1 of this study, an alternative approach for adjusting facial movement data for head motion is presented that avoids the use of a maxillary occlusal splint. For each video-

From the Departments of Orthodontics and Prosthodontics at the University of North Carolina and the Department of Statistics at the University of Michigan. Received for publication February 26, 1999; revised August 9, 1999. 
captured frame during an animation, selected facial landmarks were rotated and translated to "fit" the landmark positions of the face at rest. This approach is based on the Procrustes method. ${ }^{11}$ In part 2 of this study, our modified Procrustes method was used to adjust data for uncontrolled head motion before comparison of the maximum displacements of nasolabial landmarks among repaired unilateral and bilateral cleft lip and palate patients and noncleft control subjects. Such border positions are particularly important for evaluating cleft patients because distortions are likely to be obvious at the extremities of movements as a result of tissue restrictions (scarring) and compensatory movements of unrestricted tissues. We hypothesized that there are differences in the magnitude and asymmetry of maximum nasolabial landmark displacements among the three groups of subjects.

\section{METHODS}

\section{Instrument}

The video-based tracking system previously described (Motion Analysis, Motion Analysis Corp., Santa Rosa, Calif.) was used to track 4-mm-diameter spherical retroreflective markers attached to 30 anatomic landmarks on the face (Fig. 1). Four analog video cameras sampled the positions of these markers at 60 frames per second. Data were recorded in real time for later digitization and processing. Before recording, the head space was calibrated using a cube-shaped frame with 12 markers certified to an accuracy in position of $\pm 7.6 \mathrm{~nm}$ (Dimensional Inspection Laboratories; Fremont, Calif.). Error in specifying position with the system approximated $0.53 \mathrm{~mm}(\mathrm{SD}=0.45$ $\mathrm{mm}) .{ }^{8}$ Additional details regarding the system, calibration, data digitization, and processing can be found in Trotman et al. ${ }^{8}$

\section{Sample}

Patients were recruited from the University of Michigan Cleft Lip and Palate and Craniofacial Anomalies Program, and the noncleft subjects were recruited from students at the University of Michigan School of Dentistry. Four unilateral cleft lip and palate patients (two boys and two girls; age range, 14 to 16 years) and five bilateral cleft lip and palate patients ( 5 men, age range 13 to 21 years) were studied as well as 50 noncleft subjects (25 men and 25 women, mean age $=27.3$ years; age

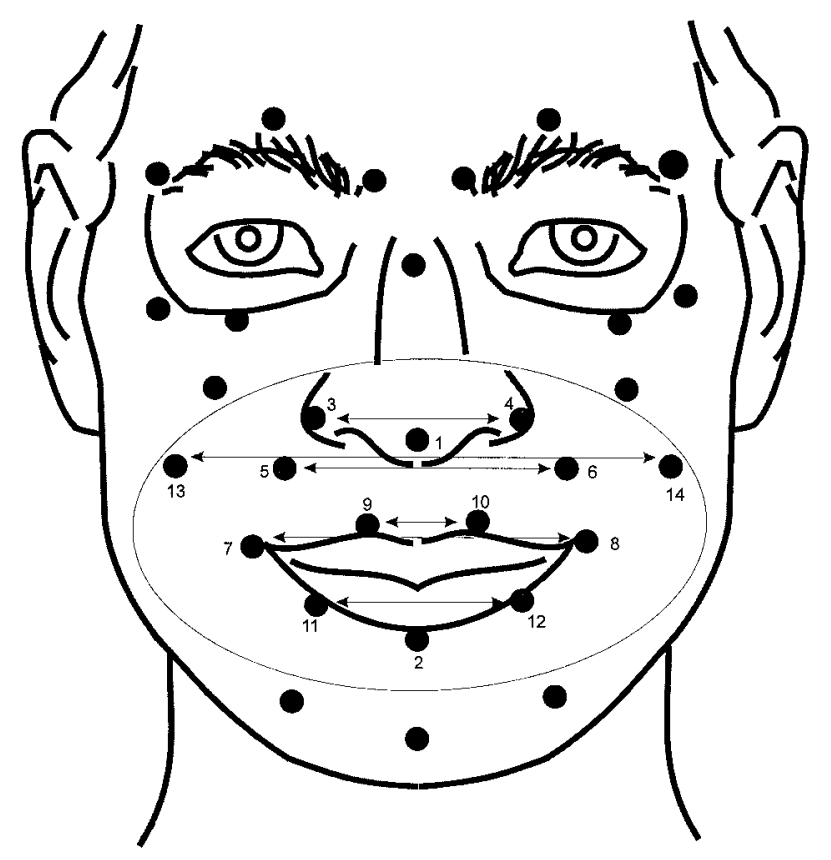

FIG. 1. All 30 facial landmarks. Those that blanket the nasolabial region and whose data are displayed in Figures 4 through 7 are enclosed within the oval. 1, nasal tip; 2, mid lower lip point located on the lower lip vermilion; 3 and 4 , right and left alar points located on the lateral alar rims; 5 and 6 , right and left nasolabial (fold) points located midway between the right and left alar rims and the right and left commissure, respectively; 7 and 8 , right and left commissure points located on the commissure; 9 and 10 , right and left upper lip points located on the peak of Cupid's bow; 11 and 12 , right and left lower lip points located on the lower lip midway between points 2 and 7 and points 2 and 8, respectively; 13 and 14, right and left outer lip points located on the cheek one-quarter of the distance between the right and left commissure and right and left temporomandibular joint regions, respectively. The six pairs from which the asymmetry scores were obtained are indicated by the double-ended arrows.

range, 23 to 39 years) who are part of a larger study for which this article serves as a preliminary report. After informed consent was obtained, the subject positioned his/her head within the calibrated space and was instructed to perform four facial animations, each three times: smile, lip purse, cheek puff, and grimace. Data were collected for 3 seconds for each of the 12 performances.

\section{Part 1: Adjustment for Head Motion}

For this part of the study, we investigated the accuracy of the modified Procrustes fit method to adjust facial movement data for naturally occurring head motion. First, for each subject and for each animation, the 10 landmarks that moved the least were identified by calculating and comparing their summed or total displacements. The movements of these 10 landmarks 
best represented the motion of the head. Second, for each frame during an animation, we rotated and translated the 10 landmarks to match their initial "rest" positions as closely as possible, minimizing the residual total squared distance. Thus, our method differs from the standard Procrustes algorithm in that only 10 of the 30 landmarks, selected on the basis of highly specific criteria, are used for matching the frames during movement and at rest.

To assess the accuracy of the modified Procrustes fit method to adjust for head motion, we compared its results to those obtained by our previous method of using a maxillary occlusal splint. For the latter, a face bow was attached to the splint and carried three reference markers. ${ }^{8,9}$ Because the markers were referenced to the cranium, their movement during the facial animations solely reflected head motion, by which the movements of soft-tissue landmarks could be adjusted.

\section{Part 2: Comparison among Cleft Patients and Con- trols}

For the second part of the study, we used the modified Procrustes fit method to adjust the facial movement data. We then compared the maximum three-dimensional displacement of 14 skin-based landmarks on the nasolabial region (Fig. 1, gray zone) of the unilateral cleft and lip palate (Fig. 2, nos. 1 to 4), the bilateral cleft lip and palate (Fig. 2, nos. 5 to 9), and the noncleft subjects. For the left unilateral patients, the images were right-left reversed to permit consistency of data interpretation among subjects. To account for facial size differences that could result in differences in landmark displacement, the distance between the right and left outer lip landmarks (Fig. 1, nos. 13 and 14) of each patient and noncleft subject was scaled to the mean for the noncleft subjects.

\section{Data Analysis and Statistics}

After adjustment for head movements, the maximum three-dimensional displacement from rest of each of the 14 landmarks was calculated for each replication of each animation and for each control subject and patient. Within-subject consistency of the maximum displacement was assessed separately for the control subjects and the patients by way of the intraclass correlation coefficient: ICC $=\sigma^{2}$ among individual $/\left(\sigma^{2}\right.$ among individual $+\sigma^{2}$ error).

Given that data were available from 50 control subjects but only 9 patients, the extent to which the values from the 9 patients were similar or different from the control data was assessed by visual inspection of plots. To determine whether the control subjects exhibited a side preference (i.e., maximum displacement on the right or left side of the face), analyses were performed on the values for the six pairs of anatomically matched landmarks (see landmarks joined by double-ended arrows in Fig. 1): right and left alar (nos. 3 and 4), right and left nasolabial fold (nos. 5 and 6), right and left

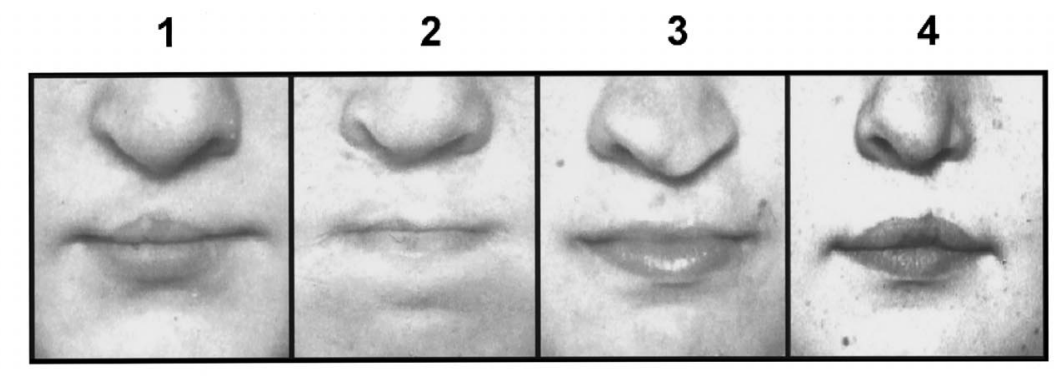

5

6

7

8

9

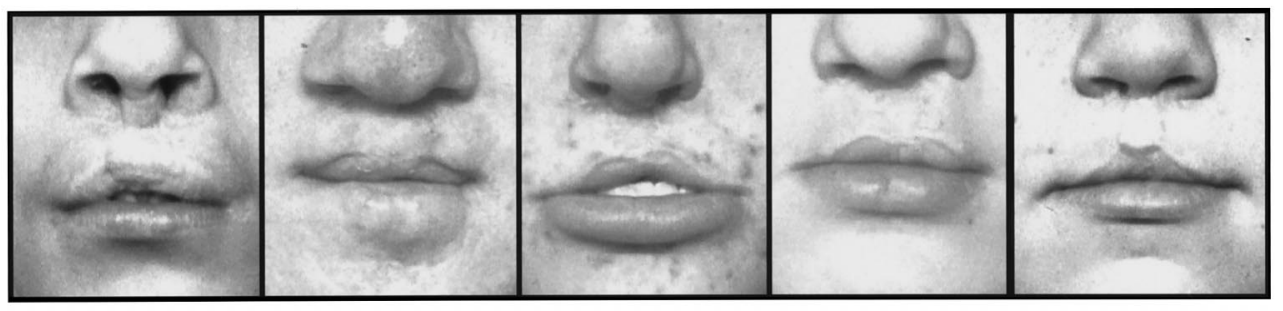

FIG. 2. Nasolabial region of unilateral (nos. 1 to 4) and bilateral (nos. 5 to 9) cleft and lip palate patients. 
commissure (nos. 7 and 8), right and left upper lip (nos. 9 and 10), right and left lower lip (nos. 11 and 12), and right and left outer lip (nos. 13 and 14). The maximum displacement values for each pair were analyzed using a mixed-model analysis of variance for effects of side and its interactions with gender and animation.

To assess the extent of asymmetry in individual subjects, we calculated a global asymmetry score by summing the absolute values of the differences in displacement over all six pairs for each subject and for each animation. Thus, if the asymmetry score is zero, the aggregate maximum displacement is exactly the same for the two sides of the face; otherwise, a positive score reflects the degree of asymmetry independently of side preference. Global asymmetry scores from the control subjects were analyzed for effects of gender, animation, and their interaction. Scores also were calculated for each of the nine patients and tabulated for comparison with the control subjects.

\section{RESULTS}

\section{Part 1: Control of Head Movement}

To illustrate our results, a mouth-opening animation in which there was substantial head movement was chosen. The motion of the landmarks with the smallest and largest movements was plotted in the top and bottom rows, respectively, of Figure 3. The movement of these two landmarks is shown under three conditions: (1) the movement based on the raw data without controlling for head motion (left column), (2) the movement after head motion was eliminated by the three stable dentitionbased reference landmark method (middle column), and (3) the movement after head motion was eliminated by the modified Procrustes fit method (right column). It can be seen that
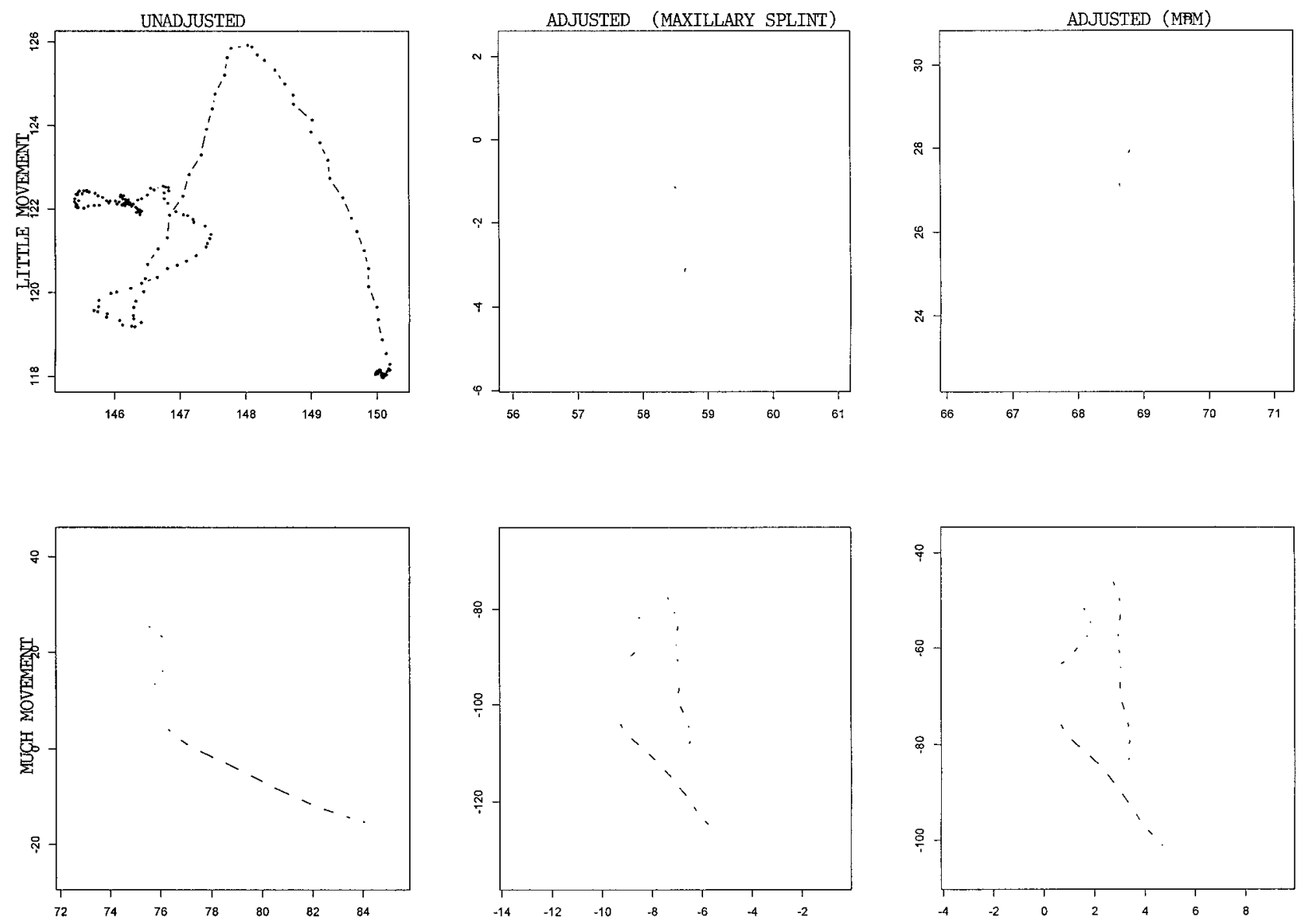

FIG. 3. Plots of the movement of the landmark with the smallest (above) and largest movements (below) during a mouthopening animation. Left column of plots, the movement based on the raw data without adjusting for head motion; middle column of plots, after head motion was eliminated by use of stable dentition-based reference landmarks; right column of plots, after head motion was eliminated by the modified Procrustes method (MPM). 
head motion was removed with both the stable reference landmark method and the Procrustes method and that the result was very similar with both methods.

\section{Part 2: Comparison among Cleft Patients and Controls}

We found the maximum displacement of the facial tissues to be reasonably consistent upon repeated performance of the animations. For the control subjects, the intraclass correlation coefficient values for each animation, averaged over landmarks, were as follows: smile, 0.68; lip purse, 0.65; cheek puff, 0.61; and grimace, 0.71 . The corresponding values for the cleft patients were as follows: smile, 0.69; lip purse, 0.67; cheek puff, 0.73; and grimace, 0.61. Although these values for the cleft and noncleft subjects were similar and averaged 0.67 , both the within- and among-variances for the cleft patients were 40 percent greater than the respective variances for the noncleft subjects.

The maximum displacements of the cleft patients were superimposed on the central 95 percent probability regions (gray boxes) of maximum displacement for the noncleft subjects (Figs. 4 through 7; see also Table I). Given this probability, it is expected that data from only 1 of the 50 control subjects would fall to the right and to the left of each box. In contrast, values from the nine cleft patients often fell below the normal range. To illustrate, maximum displacement of the right nasolabial point was notably restricted in the cleft patients during the smile, lip purse, and cheek puff animations (Figs. 4 through 6; recall that the images of the left unilateral cleft lip and palate were reversed and thus contributed to this result). For the grimace, maximum displacement of the nasolabial fold for the cleft patients approximated that of the noncleft controls, but displacement of the adjacent alar region was clearly limited. This finding reflects an emphasis of movement of the alar region during grimacing versus movement of the nasolabial point during smiling, lip pursing, and cheek puffing. It also confirms that the cleft scar affects the mobility of the nasolabial fold and alar regions, but the

Smile

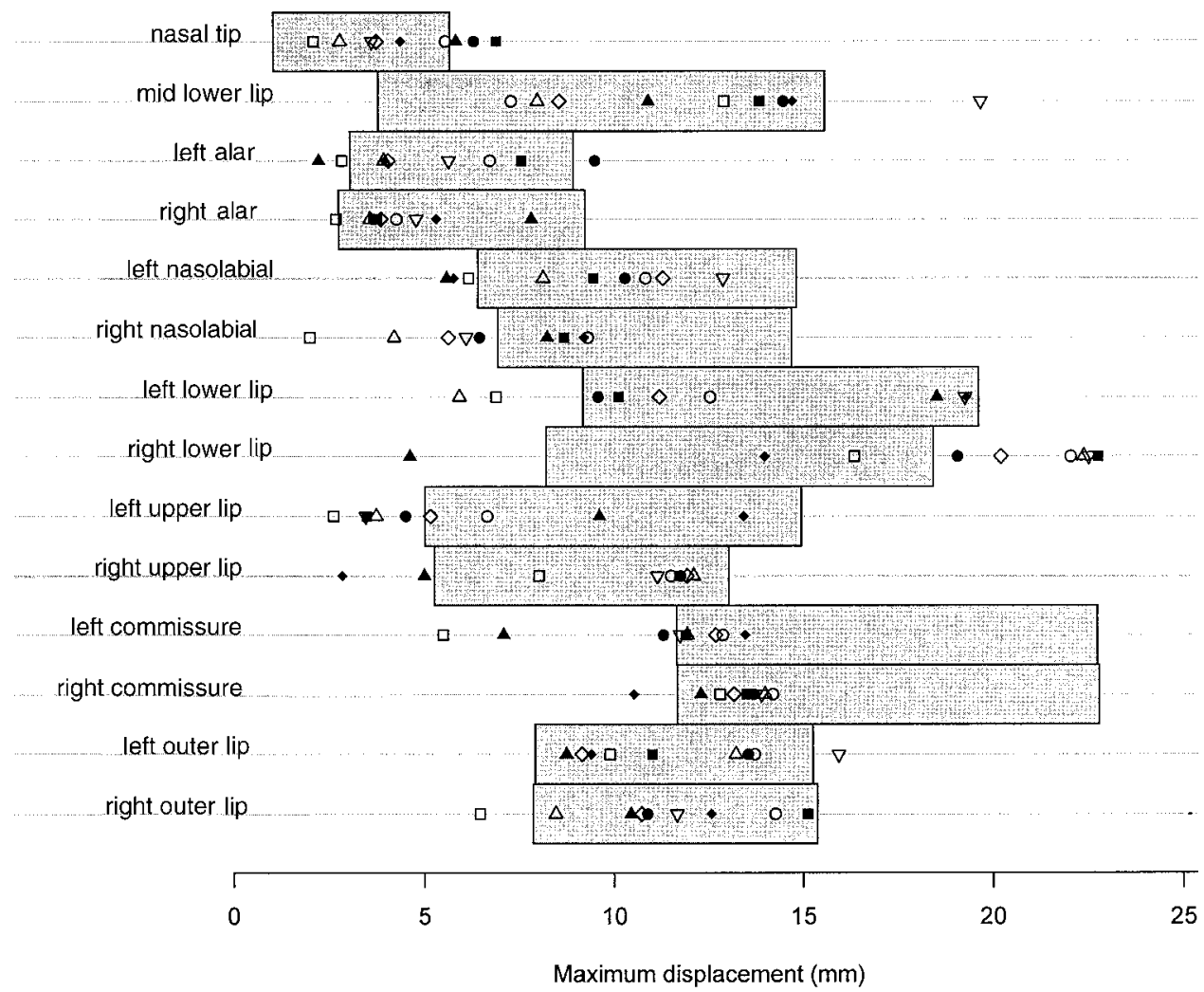

FIG. 4. Plots of the maximum three-dimensional displacement of nasolabial landmarks in the unilateral and bilateral cleft lip and palate patients during smile. The median three-dimensional displacement $(\mathrm{mm})$ of the landmarks in the cleft patients is superimposed on the central 95 percent probability regions of maximal displacement for the noncleft subjects (gray boxes). 


\section{Lip Purse}

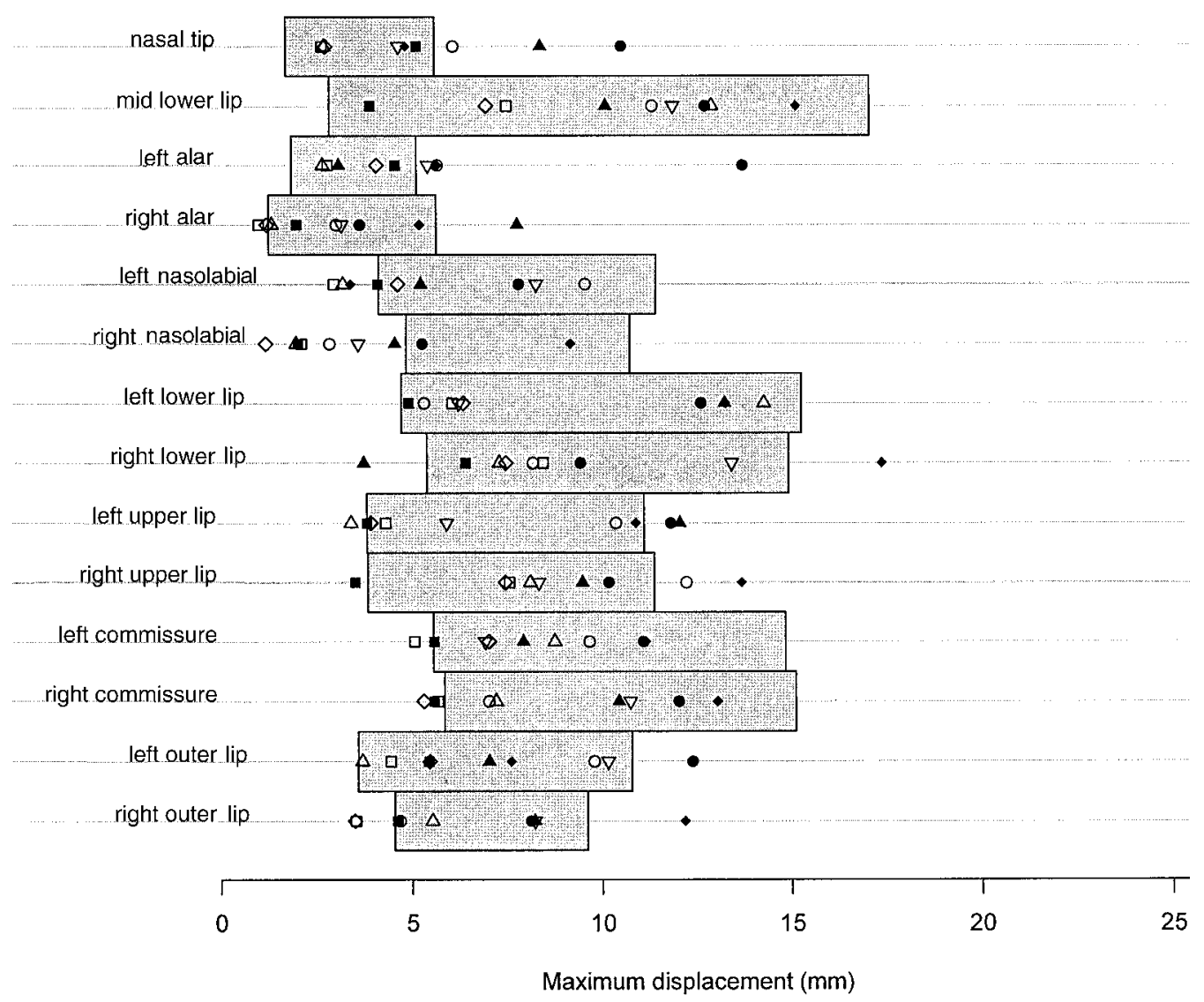

FIG. 5. Plots of the maximum three-dimensional displacement of nasolabial landmarks in unilateral and bilateral cleft lip and palate patients during lip purse. The median three-dimensional displacement $(\mathrm{mm})$ of the landmarks in the cleft patients is superimposed on the central 95 percent probability regions of maximal displacement for the noncleft subjects (gray boxes).

salience of its impact on these two regions is animation dependent.

In select cases, the maximum displacement of the cleft patients was substantially greater than normal. To illustrate, displacement of the right lower lip was exaggerated in the patients during the smile animation (Fig. 4) but not during the other three animations. Because movement of the commissure and of the right nasolabial fold region was restricted during the smile, greater movement of the right lower lip likely reflects compensatory activity to make smiling look more normal and acceptable to the cleft patients.

Analysis for a systematic side preference in the control group revealed none. On average, neither the right nor the left member of the six pairs of anatomically matched landmarks exhibited greater maximum displacement (all $p$ values in excess of 0.97 ). Individual control subjects, however, exhibited subtle degrees of asymmetry, with the right side being dominant for some and the left side for others. The individual asymmetries were most notable in the data from the right and left commissures; but even for this pair of landmarks, there was no systematic side preference across subjects of either gender for any animation.

Analysis of the global scores of asymmetry in the control group revealed a significant effect of gender $(p<0.017)$ and of the gender-byanimation interaction $(p<0.008)$. The most individual asymmetry was detected in the female smile (mean sum, $7.4 \mathrm{~mm}$ ) and the least in the female cheek puff (mean sum, $5.1 \mathrm{~mm}$ ). This result means that in the noncleft population, asymmetry in maximum displacement is likely to be most obvious in women during smiling (the right side being dominant in some women and the left side in others). The individual cleft patients, however, exhibited much greater facial asymmetry. Shown in Table II are 
Cheek Puff

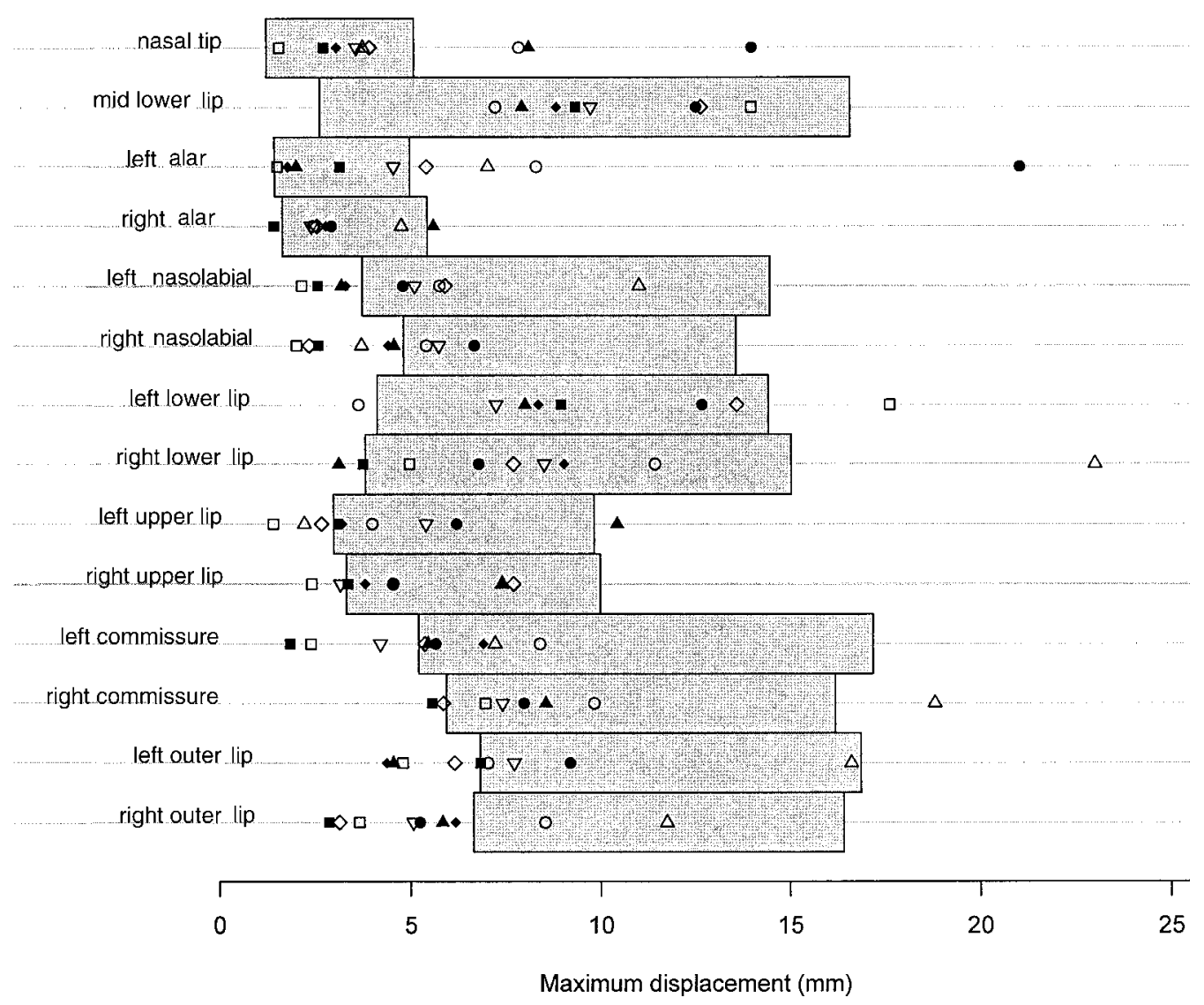

FIG. 6. Plots of the maximum three-dimensional displacement of nasolabial landmarks in the unilateral and bilateral cleft lip and palate patients during cheek puff. The median three-dimensional displacement $(\mathrm{mm})$ of the landmarks in the cleft patients is superimposed on the central 95 percent probability regions of maximal displacement for the noncleft subjects (gray boxes).

the values for each cleft patient and values for the 95 percent probability regions for the control subjects. Only 5 of the 36 values for the patients fall within the normal range of variation, and no values for the smile animation approximated normality. The differences in the control data for men and women and between the control data and patient data cannot be attributed to differences in facial size because the data were first adjusted for this source of variation.

\section{Discussion}

Using a novel method to adjust for head movements (the modified Procrustes fit method), we demonstrated differences in both the magnitude and symmetry of nasolabial displacement among cleft and noncleft subjects. Although it was tempting to describe differences among the groups (unilateral, bilateral, and noncleft) in terms of means or averages, it became clear that averages would mask the tremendous patient-to-patient variability in asymmetry and in the magnitude of displacement. These differences among patients were not surprising given the large number of factors that influence the outcome of lip surgery, such as the severity of the cleft at birth, the skill of the surgeon, the type of surgical repair, and the number of surgical revisions. ${ }^{3}$ For example, during the cheek puff animation, bilateral cleft lip and palate patient 5 demonstrated a generalized restriction in movement compared with the controls. Bilateral cleft lip and palate patient 7 , however, demonstrated highly variable maximum displacement and asymmetry. In this patient, although some landmarks had restricted movement, others had greater movement than the control subjects. This latter finding of greater movement implies that recruitment of surrounding facial regions, as well as enhanced movements, probably occurs 


\section{Grimace}

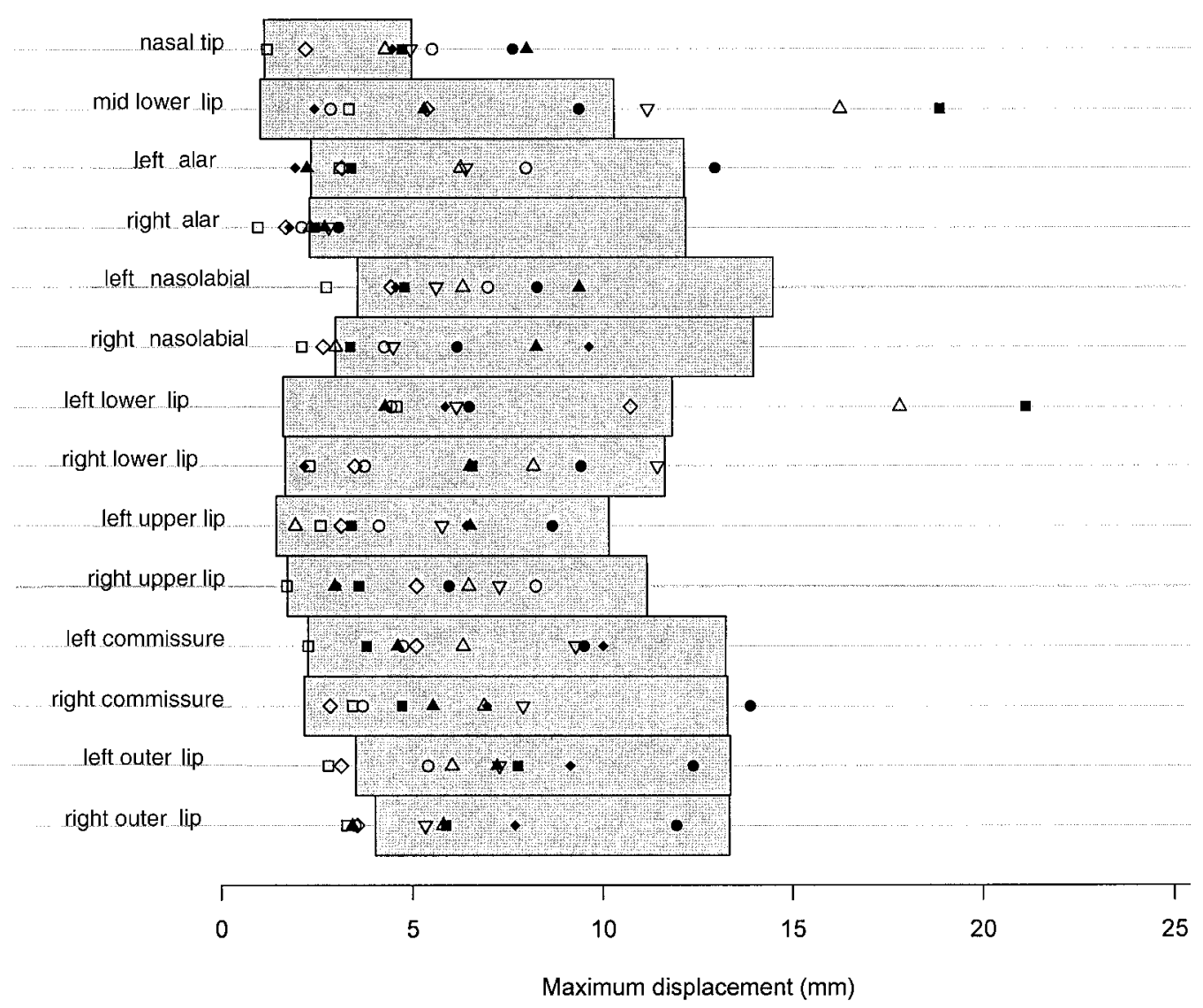

FIG. 7. Plots of the maximum three-dimensional displacement of nasolabial landmarks in unilateral and bilateral cleft lip and palate patients during grimace. The median three-dimensional displacement $(\mathrm{mm})$ of the landmarks in the cleft patients is superimposed on the central 95 percent probability regions of maximal displacement for the noncleft subjects (gray boxes).

TABLE I

Patient Profiles and Symbols for Figures 4 through 7

\begin{tabular}{clrll}
\hline Patient & \multicolumn{1}{c}{ Cleft Type } & Age & Gender & \multicolumn{1}{c}{ Symbol } \\
\hline 1 & Right unilateral & 14 & Female & Solid square \\
2 & Right unilateral & 14 & Male & Solid octagon \\
3 & Left unilateral & 16 & Female & Solid (tip-up) triangle \\
4 & Left unilateral & 14 & Male & Solid diamond \\
5 & Bilateral & 20 & Male & Clear square \\
6 & Bilateral & 21 & Male & Clear octagon \\
7 & Bilateral & 18 & Male & Clear (tip-up) triangle \\
8 & Bilateral & 15 & Male & Clear diamond \\
9 & Bilateral & 13 & Male & Clear (tip-down) triangle \\
\hline
\end{tabular}

to compensate for areas, such as the upper lip, that have restricted mobility. Additionally, the greater within- and among-subject variance for the cleft patients indicates that they were less consistent in their movements individually and as a group than the control subjects. Further, in the bilateral cleft lip and palate patients, the response to surgical lip repair was not always bilaterally symmetric; each side of the cleft in these patients seemed to function as a separate entity.

Interestingly, the noncleft subjects in our study did not demonstrate a consistent side preference in displacement for the instructed animations. This finding is in contrast with the work of others, ${ }^{12-14}$ who have reported a leftside facial dominance. There exists the possibility that a hemifacial dominance in movement could result from a hemifacial dominance in anatomic size. ${ }^{14,15}$ Differences in the size of the sides of the face in noncleft and cleft subjects have been reported. ${ }^{4-6,16-19}$ Koff and coworkers, ${ }^{15}$ however, found for most subjects that hemifacial size dominance and movement dominance were not related; the right side of the face tended to be larger whereas the left side had greater mobility (see also Hager and $\mathrm{Ekman}^{14}$ ).

Not surprisingly, we found substantially greater asymmetry in maximum displacement 
TABLE II

Asymmetry Scores for the Noncleft Subjects and for Cleft Patients 1 through 9 (see Fig. 2)

\begin{tabular}{|c|c|c|c|c|c|c|c|c|c|c|c|c|}
\hline \multirow[b]{2}{*}{ Animation } & \multicolumn{3}{|c|}{$\begin{array}{l}\text { Noncleft Subjects } \\
\quad(n=50)\end{array}$} & \multicolumn{9}{|c|}{ Cleft Patients } \\
\hline & $\begin{array}{l}\text { Lower } \\
(2.5 \%) \\
\end{array}$ & Median & $\begin{array}{l}\text { Upper } \\
(2.5 \%) \\
\end{array}$ & 1 & 2 & 3 & 4 & 5 & 6 & 7 & 8 & 9 \\
\hline Smile & 3.44 & 6.77 & 14.28 & 32.08 & 35.45 & 18.95 & 26.46 & 32.10 & 19.71 & 35.68 & 22.81 & 24.07 \\
\hline Lip purse & 2.34 & 5.32 & 16.88 & 7.25 & 24.45 & 21.79 & 19.69 & 7.29 & 21.27 & 17.50 & 16.54 & 21.49 \\
\hline Cheek puff & 1.78 & 5.63 & 13.38 & 15.93 & 32.62 & 17.70 & 8.55 & 19.83 & 19.78 & 43.34 & 19.05 & 15.19 \\
\hline Grimace & 2.06 & 5.56 & 11.32 & 20.14 & 20.79 & 12.26 & 17.35 & 8.11 & 17.87 & 23.77 & 15.60 & 14.00 \\
\hline
\end{tabular}

for cleft patients than in the control subjects. Offerman and coworkers ${ }^{3}$ reported a similar finding and noted greater movement of the lips on the cleft side than on the noncleft side in unilateral cleft lip and palate patients. In the present study, side dominance in displacement was highly variable among both the unilateral and bilateral cleft lip and palate patients as evidenced from the plots (Figs. 4 through 7). One plausible explanation for the difference in results between our study and the Offerman et al. study ${ }^{3}$ is that theirs was based on twodimensional measures, which have the potential to underestimate the magnitude of displacement on either or both sides of the face.

The implications of these findings are important to decisions regarding the need for lip-revision surgery. Surgeons generally base such decisions on the facial disfigurement at rest. Problems with movement (both restricted and exaggerated compensatory movements) are generally not considered. These results support the view that movements in these patients may be severely altered, particularly during smiling, owing to the variable impact of residual deformities and compensatory movements of surrounding facial tissues. Therefore, the assessment of facial animations should be strongly considered when contemplating lip revision surgery in these patients.

The present study also demonstrates that three-dimensional facial movement data can be adjusted, and thus corrected, for natural head movements without the need for establishing stable landmarks referenced to the cranium. This finding is most important because the use of stable references requires time- and labor-intensive construction of a maxillary bite splint for each subject, and the presence of the splint may interfere with the subject's performance of instructed animations. Alternatively, we feel that fixating the head to control for head movements results in a highly unnatural situation and in facial movements that may differ also from those observed with normal head mobility.

The procedure that we adopted for use is founded on the Procrustes adjustment. ${ }^{11}$ The basic idea is that two shapes can be made as similar as possible by rotating, translating, and rescaling one shape to match the other. Similarity is expressed by the sum of the differences (squared) in matched components, and the algorithm searches for the match between components that minimizes this residual sum. We initially considered a direct application of Procrustes rotation and translation to match video frames at the endpoint of each animation to frames obtained before its performance. The problem with this approach became readily apparent. Those landmarks that moved substantially more than others (i.e., owing to large facial tissue movement in addition to head movement) biased the match. That is, the adjustment afforded by direct application of the Procrustes method resulted in the elimination and addition of movement components to the individual markers that were clearly unrelated to head movements. In essence, a large movement in one part of the face became dispersed in data sampled from totally different areas on the face.

One solution to this problem is to downweigh the impact of the outliers (i.e., the highly mobile markers) as proposed by Verborn $^{20}$ and Dryden. ${ }^{21}$ Outliers are points that appear too far from their expected positions based on the least-squares fit of the standard Procrustes method. This solution proved to be nonoptimal for the present problem because a substantial proportion of the landmarks showed significant parallel, in-concert motion during the animations. That is, the landmarks moved in the same direction at the same velocity at the same time. As such, a large number of landmarks had to be down-weighted to accomplish a good match (small residual sum). It was particularly difficult to distinguish stable land- 
marks that moved in concert with the head from nonstable landmarks that moved in concert with the facial tissue during its displacement. In addition, the computational burden was prohibitive.

These results, however, suggested that a Procrustes fit using only a subset of markers for which movement was least might provide an acceptable match and one that is useful in adjusting the data for head movements. In this regard, Bookstein's work ${ }^{22}$ indicates that threedimensional shapes can be adequately matched using only three markers if they are relatively stable and out of line (noncollinear). A single choice of three standard markers for our purpose was unrealistic, however, because the face moves differently from animation to animation and from person to person.

Our final and adopted approach (the modified Procrustes fit method) is a compromise between those of Procrustes and Bookstein. Adjustments for head movement are based on 10 of the 30 available markers tailor-selected for each subject and animation. Instead of matching all landmarks between the beginning and end of the animation, the 10 landmarks that show the least motion (typically those in the face periphery) are matched. From our experience, use of 10 landmarks ensures sufficient lack of collinearity. Alternatively, use of more than 10 risks inclusion of landmarks with large in-concert movements and the associated problems (as discussed above). In future studies, this method will allow us to totally eliminate the occlusal splint that was key to our previous method for adjustment of head movements. Use of a splint is not well tolerated by younger patients, and the presence of a splint can be obstructive during video imaging. Given that the major distortions in facial surface contours in cleft patients are on and around the nasolabial region, the avoidance of a maxillary splint is clearly a decided advantage for studying this patient population.

Carroll-Ann Trotman, B.D.S., M.A., M.S.

Department of Orthodontics

School of Dentistry

University of North Carolina at Chapel Hill

CB\# 7450, Brauer Hall

Chapel Hill, N.C. 27599-7450

Carroll-Ann_Trotman@dentistry.unc.edu

\section{ACKNOWLEDGMENTS}

The authors thank Drs. Camilla Tulloch, William Proffit, Gerald Sloan, and Kelly B. Mitchell for helpful suggestions during the preparation of this article. This study was supported in part by National Institute of Dental Research Grant DE 05215-2151.

\section{REFERENCES}

1. Asher-McDade, C., Roberts, C., Shaw, W. C., and Gallager, C. Development of a method for rating nasolabial appearance in patients with clefts of the lip and palate. Cleft Palate Craniofac. J. 28: 385, 1991.

2. Morrant, D. G., and Shaw, W. C. Use of standardized video recordings to assess cleft surgery outcome. Cleft Palate Craniofac. J. 33: 134, 1996.

3. Offerman, R. E., Cleall, J. F., and Subtelny, J. D. Symmetry of lip activity in repaired unilateral clefts of the lip. Cleft Palate J. 1: 347, 1964.

4. Coghlan, B. A., Matthews, B., and Pigott, R. W. A computer-based method of measuring facial asymmetry: Results from an assessment of the repair of cleft deformities. Br. J. Plast. Surg. 40: 371, 1987.

5. Coghlan, B. A., Matthews, B., and Pigott, R. W. A computer-based method of measuring nasal symmetry in the cleft lip nose. Br. J. Plast. Surg. 46: 13, 1993.

6. Kyrkanides, S., Bellohusen, R., and Subtelny, J. D. Skeletal asymmetries of the nasomaxillary complex in noncleft and postsurgical unilateral cleft lip and palate individuals. Cleft Palate Craniofac. J. 32: 428, 1995.

7. Neely, J. G., Cheung, J. Y., Wood, M., Byers, J., and Rogerson, A. Computerized quantitative dynamic analysis of facial motion in the paralyzed and synkinetic face. Am. J. Otol. 13: 97, 1992.

8. Trotman, C.-A., Stohler, C. S., and Johnston, L. E., Jr. Measurement of facial soft tissue mobility in man. Cleft Palate Craniofac. J. 35: 16, 1998.

9. Trotman, C.-A., Faraway, J. J., Silvester, K. T., Greenlee, G. M., and Johnston, L. E., Jr. Sensitivity of a method for the analysis of facial mobility: I. Vector of displacement. Cleft Palate Craniofac. J. 35: 132, 1998.

10. Trotman, C.-A., and Faraway, J. J. Sensitivity of a method for the analysis of facial mobility: II. Interlandmark separation. Cleft Palate Craniofac. J. 35: 142, 1998.

11. Goodall, C. Procrustes methods in the statistical analysis of shape. J. R. Stat. Soc. Ser. B 53: 285, 1991.

12. Paletz, J. L., Manktelow, R. T., and Chaban, R. The shape of a normal smile: Implications for facial paralysis reconstruction. Plast. Reconstr. Surg. 93: 784, 1994.

13. Wood, D. A., Hughes, G. B., Secic, M., and Good, T. L. Objective measurement of normal facial movement with video microscaling. Am. J. Otol. 15: 61, 1994.

14. Hager, J. C., and Ekman, P. The Asymmetry of Facial Actions Is Inconsistent with Models of Hemispheric Specialization. In P. Ekman and E. L. Rosenberg (Eds.), What the Face Reveals. New York: Oxford University Press, 1997.

15. Koff, E., Borod, J. C., and White, B. Asymmetries for hemiface size and mobility. Neuropsychologia 19: 825, 1981.

16. Shah, S. M., and Joshi, M. R. An assessment of asymmetry in the normal craniofacial complex. Angle Orthod. 48: 141, 1978.

17. Ferrario, V. F., Sforza, C., Poggio, C. E., and Tartaglia, G. Distance from symmetry: A three-dimensional evaluation of facial asymmetry. J. Oral Maxillofac. Surg. 52: $1126,1994$. 
18. Ras, F., Habets, L. L. M. H., van Ginkel, F. C., and PrahlAndersen, B. Three-dimensional evaluation of facial asymmetry in cleft lip and palate. Cleft Palate Craniofac. J. 31: 116, 1994.

19. Burke, P. H. Stereophotogrammetric measurement of normal facial asymmetry in children. Hum. Biol. 43: 536,1971
20. Verborn, P. A Robust Approach to Nonlinear Multivariate Analysis. Leiden, The Netherlands: Leiden University DWSO Press, 1994.

21. Dryden, I. General Shape and Registration Analysis: Technical Report. Leeds, England: University of Leeds, 1998.

22. Bookstein, F. Morphometric Tools for Landmark Data. Cambridge, England: Cambridge University Press, 1991. 